
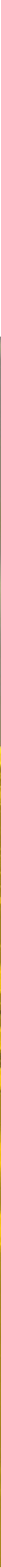
Cite this: CrystEngComm, 2013, 15, 4062

Received 23rd November 2012, Accepted 15th December 2012

DOI: $10.1039 / \mathrm{c} 2 \mathrm{ce} 26911 \mathrm{~b}$

www.rsc.org/crystengcomm

\section{Crystal growth rates and molecular dynamics of nifedipine $\dagger$}

\author{
Tanja Gnutzmann, ${ }^{a}$ Robert Kahlau, ${ }^{\text {b }}$ Stefan Scheifler, ${ }^{c}$ Ferdinand Friedrichs, ${ }^{\text {a }}$ Ernst \\ A. Rössler, ${ }^{b}$ Klaus Rademann ${ }^{\text {ac }}$ and Franziska Emmerling ${ }^{* a}$
}

\begin{abstract}
The unusually fast crystallization of the organic compound nifedipine has been investigated in detail using different solutions as precursors for the formation of the initial glassy nifedipine films. Starting from these amorphous films the crystallization process was investigated by time-resolved light microscopy at different temperatures around $T_{\mathrm{g}}$ of nifedipine. In all studied cases (acetone, acetonitrile, ethyl acetate, dichloromethane, tetrahydrofuran) the measured mean crystallization rates are, on average, 10000 times higher than rates reported in previous studies. Such high rates cannot be explained by a classical diffusional crystal growth mechanism of nifedipine. Instead, nifedipine shows a strong propensity for diffusionless growth. Solvent assisted or solvent induced preordering of the glassy modification is suggested to be the most probable driving force behind these fast crystallization processes. The nifedipine crystallization rates can be controlled not only by specific solvent-molecule interactions but also by temperature. Below $40{ }^{\circ} \mathrm{C}$, the temperature dependence of the rates is generally small. Above $40{ }^{\circ} \mathrm{C}, \mathrm{a}$ slowing down of the crystallization rates with increasing temperatures indicates a kinetic competition between different polymorphs. The molecular dynamics of nifedipine molecules in the amorphous phase as well as the relaxation times are studied by dielectric measurements. The dielectric data corroborate the inherently strong propensity of nifedipine to crystallize diffusionlessly under all conditions.
\end{abstract}

\section{Introduction}

Crystallization is one of the key processes in different areas of science covering inorganic and organic materials. Especially organic compounds are known for their polymorphism. This phenomenon characterizes the propensity of a compound to crystallize in more than one structure. ${ }^{1}$ Polymorphism directly affects solid-state properties of the materials, i.e. stability, dissolution rate of a compound and is therefore of great importance for the pharmaceutical and fine chemical industries. $^{2,3}$ The selective crystallization of a certain polymorph and its subsequent stabilization remains a challenging task, since the various thermodynamic and kinetic factors affecting the crystallization processes are difficult to control. In most cases the rate determination of the crystallization is still an experimental challenge. ${ }^{1}$ Recently, two new phenomena in the field of organic (molecular) systems crystallizing from the

${ }^{a}$ BAM Federal Institute for Materials Research and Testing, Richard-WillstaetterStrasse 11, 12489 Berlin, Germany.E-mail: franziska.emmerling@bam.de; Fax: +49308104 1137; Tel: +49308104 1133

${ }^{b}$ Institute of Physics, Universitaet Bayreuth, Bayreuth 95440, Germany

${ }^{c}$ Department of Chemistry, Humboldt Universitaet zu Berlin, Brook-Taylor-Strasse 2, 12489 Berlin, Germany. E-mail: klaus.rademann@chemie.hu-berlin.de;

Fax: +49 302093 5559; Tel: +49 3020935565

$\dagger$ Electronic supplementary information (ESI) available: Raman spectra of the crystallization experiments, Fig. S1-S6. See DOI: 10.1039/c2ce26911b melts have been described. These two phenomena i) diffusionless and ii) surface-enhanced crystallization lead to significantly higher crystallization rates than in processes driven only by bulk diffusion. ${ }^{2,3}$

Among the compounds studied in this context, nifedipine (4-(2-nitrophenyl)-2,6-dimethyl-3,5-dicarbomethoxy-1,4-dihydropyridine, see Fig. 1) is known for its polymorphism and high crystallization rates. $^{2-8}$ Nifedipine is a widely used antihypertensive and vasodilating drug of the dihydropyridine type. ${ }^{9}$ Nifedipine is perfectly suited for crystallization growth rate studies and has been intensively investigated using dielectric spectroscopy among other methods. ${ }^{10-19}$ Recently, we reported extremely high crystallization rates of nifedipine,<smiles>COC(=O)C1=C(C)NC(C)=C(C(=O)OC)C1c1ccccc1[N+](=O)[O-]</smiles>

Fig. 1 Structure of nifedipine (4-(2-nitrophenyl)-2,6-dimethyl-3,5-dicarbomethoxy-1,4-dihydropyridine). 
starting from an amorphous film produced via evaporation of an acetone solution. The crystallization process was studied by time-resolved Raman micro-spectroscopy. The mean crystallization rate of the initially crystallizing metastable $\beta$-form was found to be four orders of magnitude higher as compared to those of previous studies. The subsequent crystal conversion of the $\beta$-form to the most stable $\alpha$-form is again extremely fast. $^{8}$ Expanding systematically the acetone pilot study to different solvents ranging from very polar to non-polar, is the prime purpose of this study.

\section{Experimental section}

\section{Materials}

Nifedipine ( $\geqslant 98 \%$, CAS 21829-25-4) was purchased from Sigma Aldrich and used without further purification. On the basis of the X-ray diffraction pattern (Bruker AXS, D8, Cu K $\alpha$ radiation) and Raman spectra, the material was identified as the pure thermodynamically stable polymorph $\alpha$-nifedipine $\left(P 2_{1} / c, a=10.923(5) \AA, b=10.326(6) \AA, c=14.814(7) \AA, \beta=\right.$ $\left.92.70(6)^{\circ}, V=1669.03(494) \AA^{3}\right) .{ }^{20}$

Acetone $(\geqslant 99.5 \%)$ was purchased from Th. Geyer $\mathrm{GmbH} \&$ Co. KG (Renningen, Germany), acetonitrile ( $\geqslant 99.8 \%$ ) from J. T. Baker (Deventer, Netherlands), dichloromethane $(\geqslant 99.5 \%)$ from Carl Roth GmbH + Co. KG (Karlsruhe, Germany), chloroform $(\geqslant 99.8 \%)$ from Merck KGaA (Darmstadt, Germany), ethyl acetate $(\geqslant 99.5 \%)$ and tetrahydrofuran $(\geqslant 99.9 \%)$ from Fluka Chemie GmbH (Buchs, Switzerland).

\section{Sample preparation}

Nifedipine solutions were prepared in different solvents: acetone (182 $\mathrm{mM})$, acetonitrile (72 $\mathrm{mM})$, chloroform (202 $\mathrm{mM})$, dichloromethane (182 $\mathrm{mM})$, ethyl acetate (72 $\mathrm{mM})$, and tetrahydrofuran (200 mM). The concentration was chosen significantly below the saturation level in order to avoid the presence of any crystalline material in the starting solution. Since nifedipine is slightly light sensitive, especially in solution, any exposure to light was avoided as far as possible. The solutions were prepared at ambient conditions and stored in amber glass vials. In a typical experiment, $20 \mu \mathrm{L}$ of a freshly prepared solution of nifedipine were pipetted on a glass slide (1 mm, Menzel, Braunschweig, Germany) and left for drying in the dark for typically $1 \mathrm{~min}$. After the solvent evaporated completely, a thin glassy film of g-nifedipine remained on the surface. The thickness of the film varies with the solvent. Usually the films possess thicknesses between 50 and $70 \mu \mathrm{m}$. The surface area is around $1 \mathrm{~cm}^{2}$. Randomly distributed over the amorphous film, nifedipine started to crystallize statistically within a few minutes. The crystallization process was then monitored in situ by light microscopy and Raman spectroscopy till no more changes proceeded.

\section{Raman spectroscopy}

Raman spectroscopy was used for the characterization and the unambiguous identification of the polymorphs occurring in the thin nifedipine film. ${ }^{21}$ The Raman measurements were performed with a Raman microspectroscopic setup (LabRam,
Horiba-Jobin-Yvon, Bensheim, Germany) equipped with a BX41 microscope (Olympus, Hamburg, Germany) and a nitrogen-cooled CCD detector (256 × 1024 pixels). A HeNelaser emitting at $633 \mathrm{~nm}$ was used for excitation. To reject the Rayleigh scattered light a filter was used. The spectra were recorded with an irradiance of $1.6 \times 10^{4} \mathrm{~W} \mathrm{~cm}^{-2}$ on the sample and an acquisition time of typically $5 \mathrm{~s}$ and 5 accumulations for the ex situ spectra. All in situ spectra were recorded with an acquisition time averaging $3 \times 2 \mathrm{~s}$ or $5 \times 5 \mathrm{~s}$ adjusted to the crystal growth rate (see Fig. S1-S6, ESI $\dagger$ ).

\section{Hot stage microscopy and determination of mean crystal growth rates}

The crystallization of the thin amorphous nifedipine films was investigated in detail using light microscopy (Leica MZ 12.5, Leica Microsystems GmbH, Wetzlar, Germany, equipped with a hot stage and a $2048 \times 1536$ pixel camera). In each experiment, a thin film of nifedipine was prepared on a glass slide as described above. After the crystallization started, the propagation of one crystallization front was monitored. For each sample the growth rates of the initially formed polymorph were determined in the temperature range from $30{ }^{\circ} \mathrm{C}$ to $60{ }^{\circ} \mathrm{C}\left(55{ }^{\circ} \mathrm{C}\right.$ for nifedipine films from solution in dichloromethane). In a typical experiment a heating rate of 5 ${ }^{\circ} \mathrm{C}$ per minute was applied. At each temperature a minimum of eleven pictures were taken for the determination of the crystal growth rate. Images were taken with intervals between $1 \mathrm{~s}$ to 10 $\mathrm{s}$ adjusted to the speed of the crystal front propagation. The crystal growth rates were determined from the propagation of the crystallization front. These moving fronts are sharply defined. For each solvent at least seven samples were prepared and their crystallization processes investigated over the whole temperature range. From these repeated experiments mean crystallization growth rates and their standard deviations can be determined reproducibly.

\section{Dielectric measurements}

Dielectric measurements were carried out with an $\alpha$-A analyzer by Novocontrol, which allows for frequency resolved measurements of the dynamic susceptibility in the range of $v=10^{-2}$ $10^{6} \mathrm{~Hz}$. The sample cell is an axial symmetric parallel plate capacitor with an electrode surface of $A=2.54 \times 10^{-4} \mathrm{~m}^{2}$. The distance between the electrodes is $d=64 \mu \mathrm{m}$ determining the empty cell capacity of $C_{0}=35 \mathrm{pF}$ and the sample volume $V=$ $1.63 \times 10^{-8} \mathrm{~m}^{3}$. Thus the sample mass can be estimated to 15-20 mg. Ref. 22 gives a detailed description of the cell design. During isothermal measurements the sample temperature was kept constant within $\pm 0.2 \mathrm{~K}$ with the help of a Quatro-H temperature controller by Novocontrol. Liquid nitrogen was used as cryogenic.

\section{Results and discussion}

Thin nifedipine films were produced on a soda lime silicate glass surface by evaporation of a droplet of nifedipine solutions using six different solvents (see Fig. 2 and 5). Raman spectroscopy revealed that the thin glassy nifedipine film consisted in all cases of the amorphous form of 


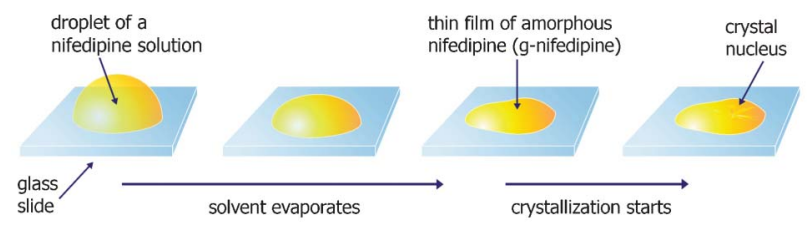

Fig. 2 Sketch of the sample preparation.

nifedipine, also denoted as g-nifedipine. ${ }^{21} \mathrm{~A}$ few minutes after the solvent evaporation, crystallization started statistically distributed over the surface of the amorphous nifedipine films. The crystallization propagated rapidly with spherulitic morphology. These general observations are independent of the specific solvent used for the production of the thin films. The crystallization process was then monitored by light microscopy. Fig. 3 displays microscopy images taken sequentially during the crystallization process of a nifedipine film produced from a solution of nifedipine in acetone. Within this frame, the images show the propagation of a crystallization front in the thin nifedipine film. The light microscope images (see Fig. 3) display the transformation of the amorphous form to the crystalline metastable $\beta$-modification of nifedipine. The assignment of the phases and their purity was carried out and verified unambiguously via Raman measurements (see e.g. Fig. 4 and text below). ${ }^{8,23,24}$

The mean crystal growth rate was determined from the propagation of the crystallization front. These moving fronts are very pronounced, spatially extended, sharply defined, and easy to follow. Therefore, reliable growth rate measurements can be conducted between an arbitrarily indicated starting line (dashed black line in Fig. 3) and the propagating front line (dashed red line in Fig. 3). The specifically chosen crystallization front in Fig. 3 propagates with $13.3 \pm 0.3 \mu \mathrm{m} \mathrm{s}^{-1}$.

Our observations presented here point to the fact that the glassy form of nifedipine, when freshly prepared from

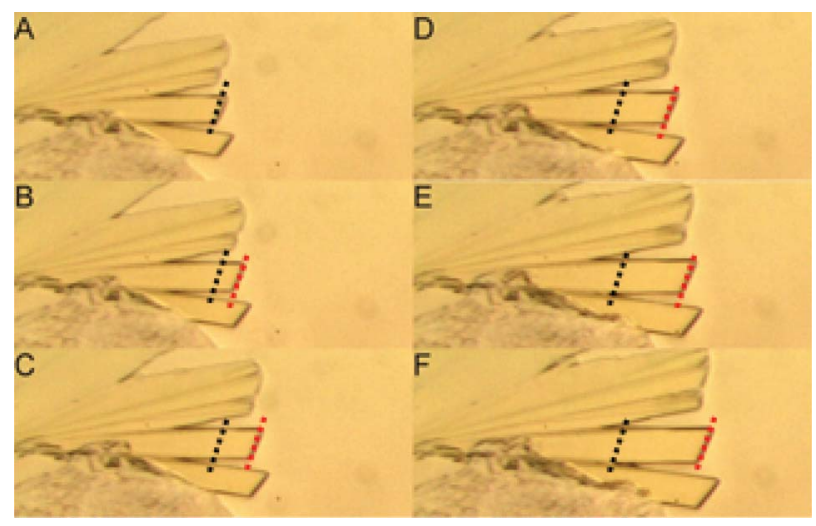

Fig. 3 Light microscope images of the crystal growth (dashed black line) of crystalline nifedipine at room temperature in a thin amorphous nifedipine film produced via evaporation of a solution in acetone $(\mathrm{A}-\mathrm{F}$, total width and height of a single image: $610 \mu \mathrm{m} \times 258 \mu \mathrm{m})$. The images are taken with an interval of $2 \mathrm{~s}$. different solutions, undergoes extremely fast crystallization in each case. This crystallization rate is not so high, when the film is calorimetrically relaxed, aged, or has a specific thermal history. Under these conditions the excess free energy value is closer to the free energy of the thermodynamically most stable polymorph and therefore more stable against crystallization. These observations and arguments are well documented for nifedipine and nifedipine alloys in the literature. ${ }^{17,18}$

The crystal growth from the amorphous films was also monitored with Raman microspectroscopy to identify the crystallizing polymorphs of nifedipine. Fig. 4 shows the Raman spectra for the crystallization of thin films produced from a solution in acetone (Fig. 4, left) and a solution in dichloromethane (Fig. 4, right). The crystallization process is followed starting with the amorphous film (bottom), which exhibits the Raman signals of amorphous g-nifedipine. For both solvents, the in situ Raman spectra show rapid crystallization to intermediate phases. In case of acetone the metastable $\beta$-modification was formed from the amorphous precursor after $16 \mathrm{~s}$. For dichloromethane the Raman spectra show features that could not be assigned to the known modifications of nifedipine as described by Chan et al. ${ }^{21}$ For both solvents the crystallization of the intermediate phase is followed by a transformation to the thermodynamically stable $\alpha$-modification (top).

The assignment of the different modifications is unambiguous by the characteristic C-C-O-vibration of the ester groups at $1215 \mathrm{~cm}^{-1}(\mathrm{~g}, \beta)$ and $1225 \mathrm{~cm}^{-1}(\alpha)$, the symmetric stretching vibration of the $\mathrm{NO}_{2}$-group at $1355 \mathrm{~cm}^{-1}(\mathrm{~g})$ and $1348 \mathrm{~cm}^{-1}(\alpha, \beta)$ as well as the $\mathrm{C}=\mathrm{C}$ stretching vibration at 1652 $\mathrm{cm}^{-1}(\beta)$, respectively, $1648 \mathrm{~cm}^{-1}(\mathrm{~g}, \alpha)$. Furthermore, the ester bond stretching modes in the wave number region between 1660 and $1710 \mathrm{~cm}^{-1}$ can be used for differentiation. The $\mathrm{C}=\mathrm{O}$ stretching vibration at $1680 \mathrm{~cm}^{-1}$ with high relative intensity is characteristic for the $\alpha$-modification while the spectra of the $\beta$-form display signals at $1663 \mathrm{~cm}^{-1}, 1675 \mathrm{~cm}^{-1}$ and 1701 $\mathrm{cm}^{-1} \cdot{ }^{21}$ The amorphous g-nifedipine is characterized by broader peaks compared to the crystalline material.

The Raman spectra of the intermediate phase observed for the crystallization of the thin films from dichloromethane (see Fig. 4, right) show only slight differences when compared to the spectra of the known nifedipine polymorphs. Regarding the characteristic signals of the different nifedipine polymorphs, the new polymorph was distinguished from the other modifications by signals at $1214 \mathrm{~cm}^{-1}, 1364 \mathrm{~cm}^{-1}, 1638 \mathrm{~cm}^{-1}$, $1655 \mathrm{~cm}^{-1}$ and $1703 \mathrm{~cm}^{-1}$. Therefore we presume that this might be a new polymorph of nifedipine. Structural analysis of this compound is in progress.

The mean crystal growth rates of the first crystallizing phase were determined from at least seven repeated experiments as a function of temperature depending on the different solvents. Fig. 5 displays the mean crystal growth rates with their respective standard deviation for the transformation of the amorphous material to the initially crystallizing polymorphic modification. For all solvents the resulting amor- 

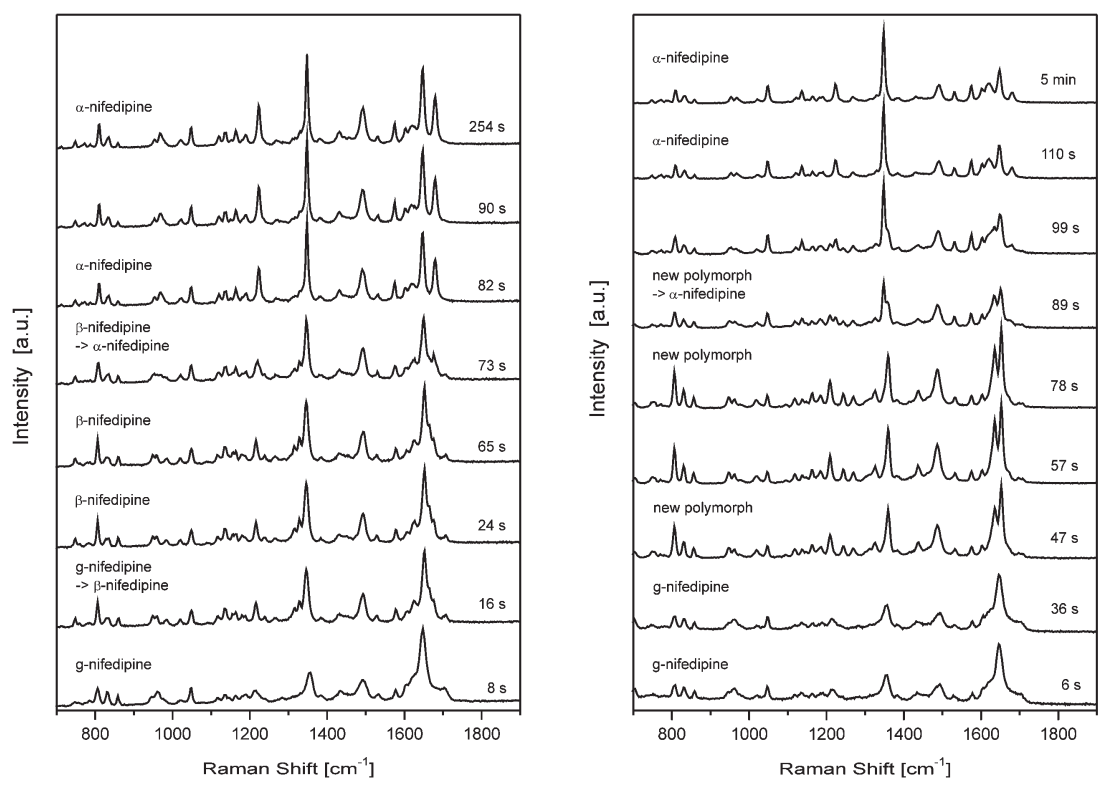

Fig. 4 Time-resolved Raman spectra of nifedipine samples measured during the growth of the crystallites from the thin films produced from a solution in acetone (left), and in dichloromethane (right) starting with the amorphous film (bottom) to the thermodynamically stable crystalline material (top). The spectra show the rapid evolution of intermediate phases in case for acetone $\beta$-nifedipine (24 s). For dichloromethane, a new nifedipine modification ( $47 \mathrm{~s}$ ) and the subsequent conversion to the $\alpha$-nifedipine (at $73 \mathrm{~s}$ for acetone and $89 \mathrm{~s}$ to $110 \mathrm{~s}$ for dichloromethane). Note the transition to the stable $\alpha$ phase occurs at times longer than $73 \mathrm{~s}$.
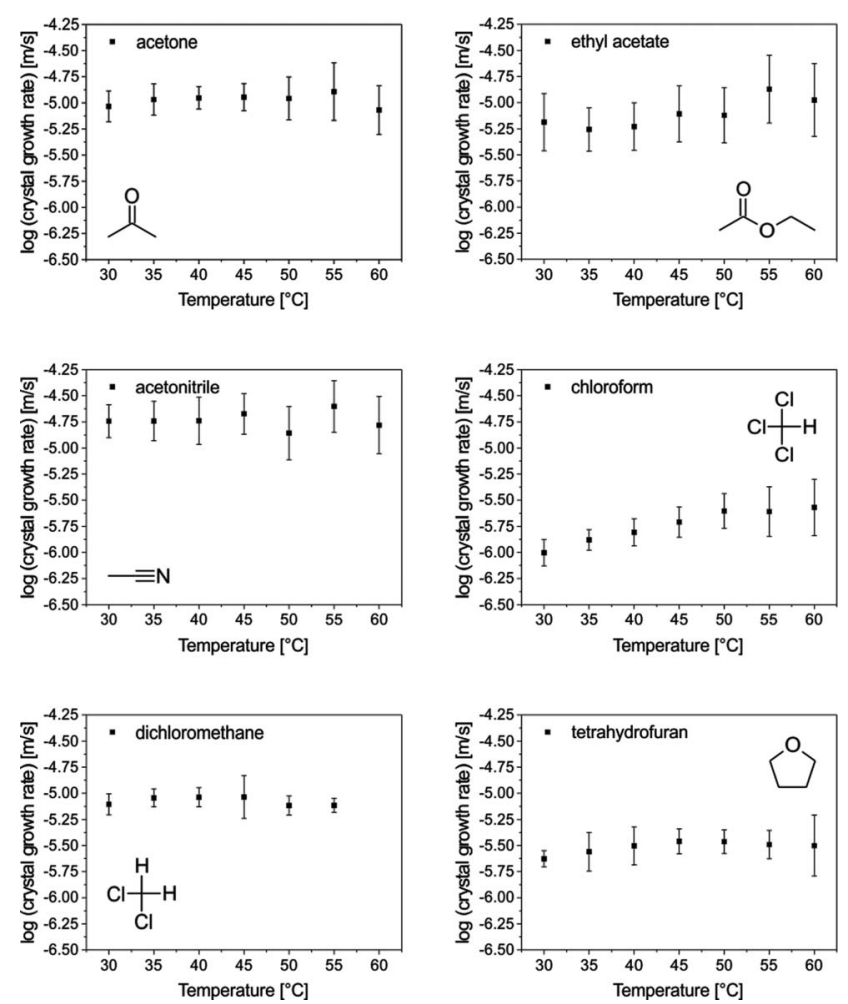

Fig. 5 Mean crystal growth rates (symbols) of nifedipine crystals from an amorphous film produced from solutions in different solvents. The extension of the bars reflects the results of several repeated experiments. The crystal growth rates are shown as a function of temperature in steps of $5^{\circ} \mathrm{C}$. phous nifedipine film crystallized unusually fast. The crystal growth rates are in the order of $10 \mu \mathrm{m} \mathrm{s}^{-1}$.

\section{Acetone}

The crystal growth rates for the thin amorphous nifedipine films obtained from a solution in acetone were in the range of $9 \mu \mathrm{m} \mathrm{s}^{-1}$ to $13 \mu \mathrm{m} \mathrm{s}^{-1}$. This is in accordance with previously published data. ${ }^{8}$ The crystal growth rates increase when the temperature increases from $30{ }^{\circ} \mathrm{C}$ to $45{ }^{\circ} \mathrm{C}$. The rates stagnate or even decrease when the temperature is between $50{ }^{\circ} \mathrm{C}$ to 60 ${ }^{\circ} \mathrm{C}$. The thin film from acetone revealed only the Raman signals of the amorphous g-nifedipine. Signals of the solvent could not be detected (see Fig. S1, ESI $\dagger$ ). The amorphous film crystallized to the $\beta$-modification which transformed to the stable $\alpha$-nifedipine, as displayed in Fig. 4.

\section{Ethyl acetate}

The crystallization of a thin film from a solution in ethyl acetate proceeds with $6.5 \mu \mathrm{m} \mathrm{s}^{-1}$ at $30{ }^{\circ} \mathrm{C}$, and then decreases to $5.5 \mu \mathrm{m} \mathrm{s}^{-1}$ at $35{ }^{\circ} \mathrm{C}$. Subsequently, the growth rate increases with temperature to $13.5 \mu \mathrm{m} \mathrm{s}^{-1}$ at $55{ }^{\circ} \mathrm{C}$. The Raman spectra of the thin films can be assigned to the amorphous form of nifedipine, without contributions of the solvent (see Fig. S1, ESI ${ }^{\dagger}$. The initial crystal nuclei form very fast yielding transparent, almost colourless spherulites which soon stop growing. The crystallization then restarts from these nuclei with slower crystal growth rate. The Raman spectra of the white nuclei revealed the features of a new polymorph of nifedipine as a pure phase (see Fig. S2, ESI $\dagger$ ). The crystallites formed during the slower second crystallization process were identified as a mixture of $\beta$-nifedipine and the new polymorph (see Fig. S3, ESI†). 


\section{Acetonitrile}

The crystal growth rates of amorphous films produced from a solution of nifedipine in acetonitrile are in the range between $18 \mu \mathrm{m} \mathrm{s}^{-1}$ to $25 \mu \mathrm{m} \mathrm{s}^{-1}$. The standard deviation is very high and a dependence of the crystal growth rate on the temperature is not clearly detectable. For a given temperature the variation in the crystal growth rates is large. Nevertheless the mean crystal growth rate of the films from acetonitrile is the highest amongst all investigated solvents. The Raman spectra of the amorphous film produced from a solution of nifedipine in acetonitrile show the features of the amorphous form and also small contributions of the solvent at $2253 \mathrm{~cm}^{-1}$ (see Fig. S1, ESI $\dagger$ ). Time-resolved Raman spectra collected during the crystallization process revealed a direct crystallization of the amorphous form to the thermodynamically stable $\alpha$-modification (see Fig. S4, ESI $\dagger$ ).

\section{Chloroform}

The crystallization of amorphous nifedipine films produced from a solution in chloroform is rather slow as compared to the crystallization from thin films produced from the other solvents. The crystal growth rate rises from $1 \mu \mathrm{m} \mathrm{s}^{-1}$ at $30{ }^{\circ} \mathrm{C}$ to $2.7 \mu \mathrm{m} \mathrm{s}^{-1}$ at $60{ }^{\circ} \mathrm{C}$ starting with a fast increase in velocity up to $50{ }^{\circ} \mathrm{C}$ followed by slightly slower crystal growth rates. The Raman spectra of the thin films can be assigned to the amorphous g-nifedipine, but reveal also the three most intense signals of chloroform at 263, 368 and $668 \mathrm{~cm}^{-1}$ (see Fig. S1, ESI $\dagger$ ). The Raman spectra of the growing spherulites can be assigned to the new polymorph of nifedipine whereas the spectra directly at the centre of the spherulites show contributions from g-nifedipine, the new polymorph of nifedipine and $\alpha$-nifedipine (see Fig. S5, ESI $\dagger$ ).

\section{Dichloromethane}

For thin films from a solution in dichloromethane a slight increase of the crystal growth rates with rising temperatures was observed starting from $8 \mu \mathrm{m} \mathrm{s}^{-1}$ at $30{ }^{\circ} \mathrm{C}$ to $9 \mu \mathrm{m} \mathrm{s}{ }^{-1}$ at 45 ${ }^{\circ} \mathrm{C}$. At $50{ }^{\circ} \mathrm{C}$ and $55{ }^{\circ} \mathrm{C}$ the growth rate dropped to $7.6 \mu \mathrm{m} \mathrm{s}^{-1}$. The Raman spectra of the amorphous film exhibit the signals of the amorphous film with a fraction of remaining solvent, verified by the signals at $284 \mathrm{~cm}^{-1}$ and $702 \mathrm{~cm}^{-1}$ (see Fig. S1, ESI $\dagger$ ). In situ Raman spectroscopy revealed the transformation of the amorphous film to the final thermodynamically stable crystalline product $\alpha$-nifedipine. Half of the conducted crystallization experiments yielded directly the $\alpha$-modification from the amorphous precursor. In the other cases the formation of an intermediate phase could be detected. The Raman spectra showed the rapid crystallization of the new polymorph and subsequent transformation to the stable $\alpha$-nifedipine within $40 \mathrm{~s}$ to $60 \mathrm{~s}$, see Fig. 4, right.

\section{Tetrahydrofuran}

Thin amorphous nifedipine films from a solution in tetrahydrofuran crystallize with growth rates between $2.4 \mu \mathrm{m} \mathrm{s}^{-1}$ at $30{ }^{\circ} \mathrm{C}$ and a maximum of $3.5 \mu \mathrm{m} \mathrm{s}^{-1}$ at $45{ }^{\circ} \mathrm{C}$. With rising temperatures the growth rates exhibit an increase up to $45{ }^{\circ} \mathrm{C}$ followed by a slight decrease of the growth rates down to 3.2 $\mu \mathrm{m} \mathrm{s}{ }^{-1}$ at $60{ }^{\circ} \mathrm{C}$. The thin amorphous films contain small amounts of solvent as proven by an additional signal at 914 $\mathrm{cm}^{-1}$ in the Raman spectra of g-nifedipine (see Fig. S1, ESI $\dagger$ ). The crystallization was monitored by time-resolved Raman spectroscopy. The amorphous film crystallizes in nearly transparent/colourless spherulites. Again the Raman spectra show the features of a new polymorph of nifedipine (see Fig. $\mathrm{S} 6, \mathrm{ESI}+$ ). The new form is at least stable for $30 \mathrm{~min}$, but at the center of the spherulites the Raman spectra reveal already the transformation to the thermodynamically stable form $\alpha$-nifedipine. All Raman spectra of the crystallites of the new nifedipine form still show the most intense signal from tetrahydrofuran at $914 \mathrm{~cm}^{-1}$ also in the $\alpha$-form at the nucleus.

The newly determined mean crystal growth rates derived for the thin films from different solutions are displayed in Fig. 6 together with the growth rates reported in the literature for the supercooled melt of bulk nifedipine. Zhu et al. investigated the crystallization of nifedipine crystals in the bulk, on the surface of a nifedipine layer and on a gold coated nifedipine surface. ${ }^{3}$ They observed high crystal growth rates on the surface of the uncoated nifedipine material and interpreted this phenomenon as surface-enhanced diffusionless crystal growth. The growth rates from the thin nifedipine films produced by evaporation of a nifedipine solution in different solvents are reproducibly three to four orders of magnitude higher. These high growth rates can only be explained in terms of diffusionless crystal growth.

Since dielectric spectroscopy is a convenient technique for studying molecular dynamics in a very accurate way, ${ }^{25-27}$ we applied the method to nifedipine. As a first step we investigated the dielectric relaxation features of the supercooled liquid in order to determine the structural relaxation times $\tau_{\alpha}$, which are expected to control the crystallization process. Fig. 7a shows selected dielectric susceptibility spectra

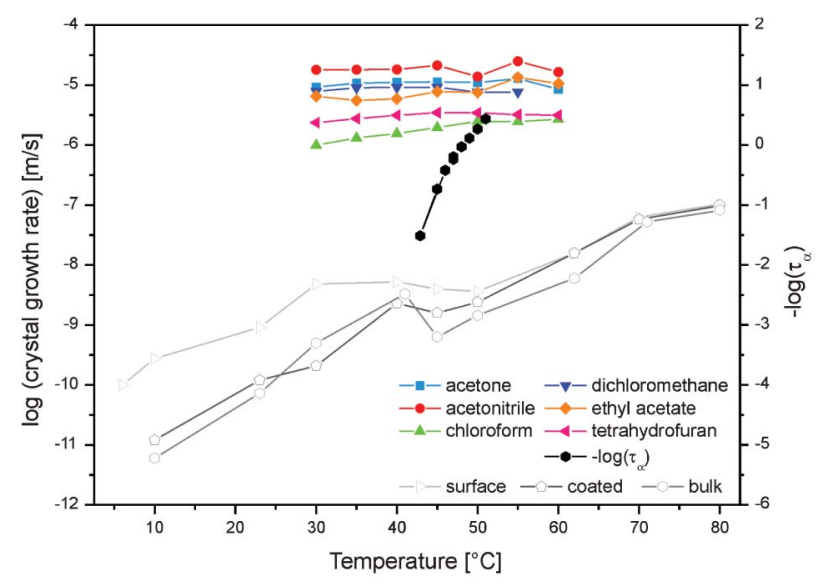

Fig. 6 The crystal growth rates derived for the crystallization from thin nifedipine films as a function of the temperature for the various solutions used to prepare the amorphous films. The data are displayed in comparison to the crystal growth rates from the supercooled melt. The black symbols show the time constant of the primary relaxation $\tau_{\alpha}(T)$ derived from dielectric measurements. The grey data points with open symbols have been reproduced from the literature. ${ }^{3}$ These data points reflect the crystallization of bulk crystals, crystals grown on the surface of bulk nifedipine, and crystals grown on gold coated surfaces of bulk nifedipine. 

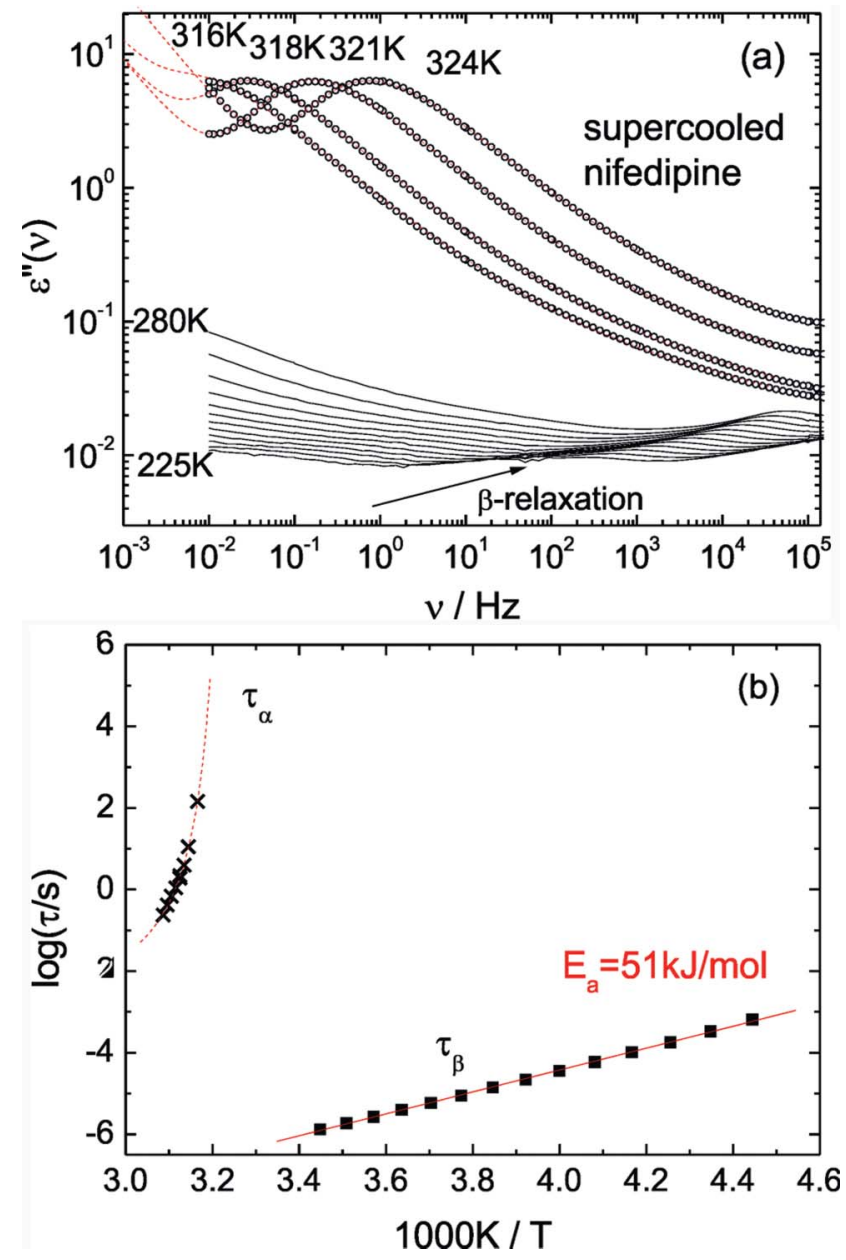

Fig. 7 (a) Dielectric susceptibility spectra of nifedipine above (symbols) and below $T_{\mathrm{g}}$ (solid lines) at indicated temperatures ( $5 \mathrm{~K}$ steps in the range of $225-$ $280 \mathrm{~K}$. A small loss of signal amplitude due to partial sample crystallization above $T_{\mathrm{g}}$ was compensated here by scaling the high- $T$ data to the amplitude of the $318 \mathrm{~K}$ curve). Dashed lines: interpolations by a phenomenological model as described in the text. (b) Corresponding relaxation times $\tau_{\alpha}$ (crosses) calculated from the fit parameters and $\tau_{\beta}$ (squares, inverse peak frequencies). Solid line: Arrhenius interpolation (activation energy indicated). Dashed line: interpolation by a Vogel-Fulcher-Tammann equation.

(black lines) clearly displaying the main relaxation $(\alpha$-)peak shifting to lower temperatures upon cooling. In addition, a socalled excess wing is found as an additional power law contribution on the high frequency side of the main relaxation peak as is typical for many molecular glass formers. ${ }^{28}$ Below $T_{\mathrm{g}}$ a very weak secondary relaxation process is identified. As shown in ref. 29 and 30 the excess wing contribution can be included in a phenomenological interpolation of the spectra (dashed line in Fig. 7a, also including a conductivity contribution). The analysis yields the time constants $\tau_{\alpha}(T)$ (cf. Fig. 7b), which agree well with those reported in the literature. ${ }^{13}$ By tracking the frequency positions of the $\beta$-peak we determined its time constants via $\tau_{\beta}=\left(2 \pi v_{\max }\right)^{-1}$ and included them in Fig. $7 \mathrm{~b}$. The $\tau_{\beta}$ follow an Arrhenius temperature dependence with an activation energy $E_{\mathrm{a}}=51 \mathrm{~kJ}$ $\mathrm{mol}^{-1}$. In contrast to that, the $\alpha$-process exhibits a super-
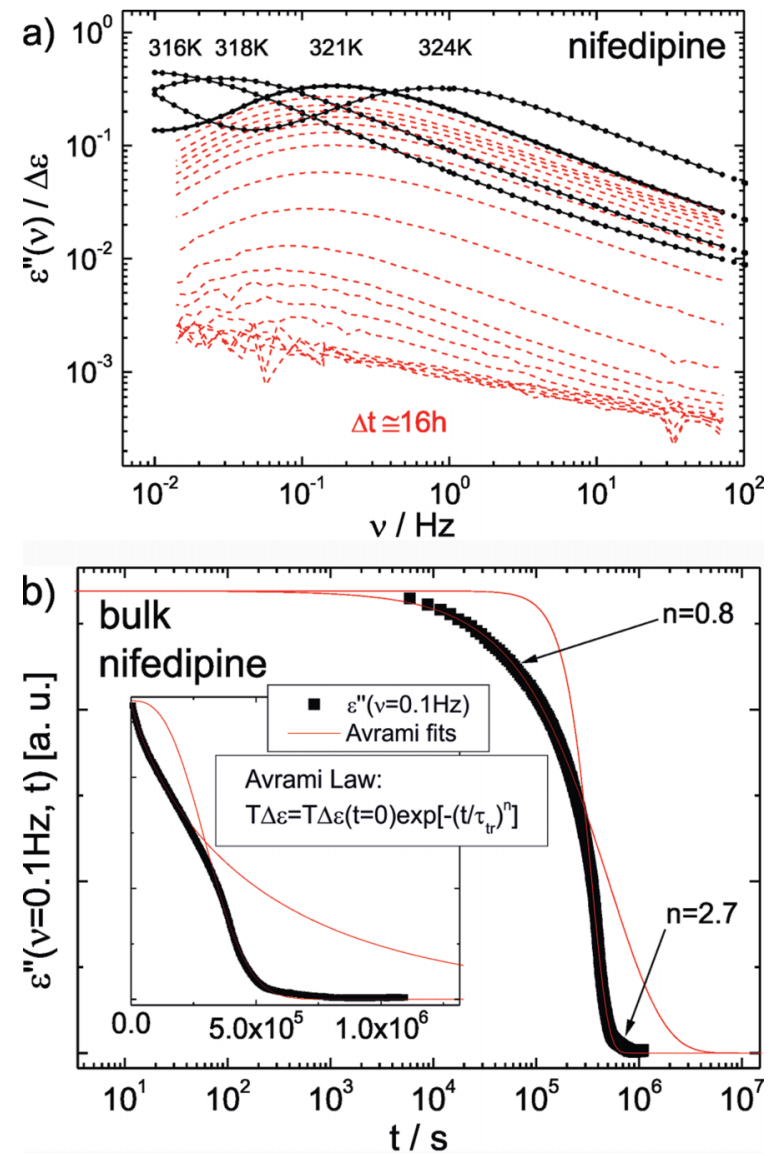

Fig. 8 (a) Dielectric spectra of nifedipine at indicated temperatures (data from Fig. 7a, connected symbols). Dashed lines: spectra at $321 \mathrm{~K}$ at different measurement times (cf. text above). (b) Spectral amplitude at constant frequency $(v=0.1 \mathrm{~Hz})$ and temperature $(T=321 \mathrm{~K})$ plotted versus logarithmic measurement time (squares). Solid lines: piece-wise interpolations with two Avrami laws (exponents indicated). Inset: the same data with linear time abscissa.

Arrhenius temperature dependence, as is typically found for supercooled liquids. The glass transition temperature is determined to $T_{\mathrm{g}}=T\left(\tau_{\alpha}=100 \mathrm{~s}\right)=316 \mathrm{~K}$. For comparison the $\tau_{\alpha}(T)$ are also included in Fig. 6 (black dots). The much stronger temperature dependence of the $\tau_{\alpha}$ compared to the one of the crystal growth rates indicates the decoupling of the crystallization kinetics from the mechanism of diffusion, as already discussed.

The transition from the highly viscous liquid to a crystalline phase, the latter displaying a very weak dielectric loss, can be monitored in a time resolved way by re-measuring the dielectric spectra in constant time intervals. In Fig. 8a we show the phase transition at $321 \mathrm{~K}$ (close to $T_{\mathrm{g}}$ ) with a time increment of about $16 \mathrm{~h}$, i.e. the total transformation covered roughly $10^{6} \mathrm{~s}$. In Fig. $8 \mathrm{~b}$ we present the time dependence of the amplitude of the susceptibility at constant $\tau=0.1 \mathrm{~Hz}$. Clearly, non-exponential transformation kinetics is observed. Displaying the data on linear time scale (inset) one recognizes a bimodal decay which can be approximated by two Avrami decays with respective exponents $n$ ( $c f$. inset Fig. $8 \mathrm{~b}$ ). The slow 
initial decay described with $n=0.8$ bends over into a much faster decay with $n=2.7$ after about $5 \times 10^{5} \mathrm{~s}$. However, it is not easy to unambiguously attribute the two decays to diffusion controlled or diffusionless crystal growth, respectively. Analyzing the produced crystalline sample after full transformation by XRD we identified the $\alpha$-modification of nifedipine. Still it may be possible that the initial slow decay reflects the transformation of the highly supercooled liquid to a metastable modification. Attempting to monitor the bimodal kinetics at higher temperatures we only observed a monomodal transformation. We note that we were able to observe crystallization even below $T_{\mathrm{g}}$. However, due to the fact that at such temperatures the main relaxation process is situated beyond the accessible frequency range and the emerging crystalline phase shows a very weak dielectric signal, we cannot monitor the transformation process quantitatively. Nevertheless, this may indicate the occurrence of a fast (diffusionless) mode of crystallization. The experimental identification of the phase formed during the first stage appears not to be directly possible. Only a combination with other experimental techniques may answer these open questions.

The different solvents used for the formation of amorphous films have a specific influence on the formation of the crystalline phase. We observed that the initially formed crystalline phase can be $\alpha$-nifedipine (acetonitrile, dichloromethane), $\beta$-nifedipine (acetone) or a novel polymorphic phase of nifedipine (ethyl acetate, chloroform, dichloromethane, tetrahydrofuran). This shows that the solvent directs selectively the first formed crystalline polymorph. It is therefore reasonable to assume that the solvent-solute interactions lead to a specific self-assembly of the nifedipine molecules in the amorphous film. This kind of preordering might also be the reason for the unusually high crystallization rates. Nifedipine self-association is a prerequisite for the very fast i.e. diffusionless crystallization. In some cases, traces of the solvents remain in the amorphous films. The presence of these traces does not show any distinct influence on the crystal growth rates. Hence the self-association and preordering of the nifedipine molecules in the amorphous films seems to be very strong. In addition to these observations, it is remarkable that different solvents have the capability to trigger the form of the initially crystallizing polymorph. For all investigated solvents, the crystal growth rates for the transformation from the amorphous films to the first crystalline phase increase with temperature up to $45^{\circ} \mathrm{C}$. A further increase of the temperature does not result in higher growth rates but stagnation or even a slight decrease of the growth rates is observed. Displayed standard deviations for the growth rates are quite large, especially for higher temperatures. This general phenomenon might be an indication for the competing crystallization of another polymorph starting at higher temperatures.

\section{Conclusions}

We conclude that nifedipine crystallizes with a strong diffusionless propensity. Observing three different solid forms (glassy $\mathrm{g}$-form, $\beta$-form, $\alpha$-form) of nifedipine within less than ten minutes is remarkable. Different solvents yield amorphous nifedipine films with different crystal growth rates, but the crystallization process as such is always fast. Crystallisation experiments at elevated temperatures (around $45{ }^{\circ} \mathrm{C}$ up to 60 $\left.{ }^{\circ} \mathrm{C}\right)$ do not lead to higher crystallization rates. Instead a stagnation or even decrease of the crystallization rate is observed. This unexpected behaviour is tentatively interpreted in terms of a competing crystallization process of a novel polymorph.

The growth rates are four orders of magnitude higher as compared to crystal growth rates driven solely by diffusion. A crystallization mechanism based on the self-association and preordering of the molecules in the freshly formed amorphous state is suggested. These findings and observations may also be applicable to other organic compounds with a $T_{\mathrm{g}}$ around room temperature.

The phenomenon of rapid crystallization from amorphous films is of importance with respect to pharmaceutical applications. Poorly soluble active pharmaceutical ingredients are sometimes formulated and marketed in their amorphous form. Hence, the stability of the amorphous form against crystallization and the possibility of competitive fast crystallization in solution, and on films on surfaces need to be characterized in a broader temperature range in order to unravel the basic underlying fast crystallization mechanisms.

\section{Acknowledgements}

We gratefully acknowledge financial support by the Deutsche Forschungsgemeinschaft through SPP 1415 (Crystalline nonequilibrium phases): Ra494/15-1, Em198/4-1, and Ro907/14.

\section{References}

1 S. A. Kulkarni, E. S. McGarrity, H. Meekes and J. H. ter Horst, Chem. Commun., 2012, 48, 4983.

2 Y. Sun, H. M. Xi, S. Chen, M. D. Ediger and L. Yu, J. Phys. Chem. B, 2008, 112, 5594.

3 L. Zhu, L. Wong and L. Yu, Mol. Pharmaceutics, 2008, 5, 921.

4 Y. Sun, L. Zhu, K. L. Kearns, M. D. Ediger and L. Yu, Proc. Natl. Acad. Sci. U. S. A., 2011, 108, 5990.

5 L. Zhu, C. W. Brian, S. F. Swallen, P. T. Straus, M. D. Ediger and L. Yu, Phys. Rev. Lett., 2011, 106, 256103.

6 Y. Sun, H. Xi, M. D. Ediger and L. Yu, J. Phys. Chem. B, 2008, 112, 661.

7 Y. Sun, H. Xi, M. D. Ediger, R. Richert and L. Yu, J. Chem. Phys., 2009, 131, 074506.

8 T. Gnutzmann, K. Rademann and F. Emmerling, Chem. Commun., 2012, 48, 1638.

9 W. Vater, K. Schlossmann, K. Stoepel, F. Hoffmeister, G. Kroneberg, W. Puls, H. Kaller, A. Oberdorf and K. Meng, Arzneim.-Forsch., 1972, 22, 1.

10 C. Bhugra, R. Shmeis, S. L. Krill and M. J. Pikal, Pharm. Res., 2006, 23, 2277.

11 T. El Goresy and R. Boehmer, J. Non-Cryst. Solids, 2006, 352, 4459. 
12 C. Bhugra, S. Rambhatla, A. Bakri, S. P. Duddu, D. P. Miller, M. J. Pikal and D. Lechuga-Ballesteros, J. Pharm. Sci., 2007, 96, 1258.

13 T. El Goresy and R. Boehmer, J. Phys.: Condens. Matter, 2007, 19, 205134.

14 D. Zhou, D. J. W. Grant, G. G. Z. Zhang, D. Law and E. A. Schmitt, J. Pharm. Sci., 2007, 96, 71.

15 C. Bhugra, R. Shmeis, S. L. Krill and M. J. Pikal, J. Pharm. Sci., 2008, 97, 4498.

16 C. Bhugra, R. Shmeis, S. L. Krill and M. J. Pikal, J. Pharm. Sci., 2008, 97, 455.

17 G. P. Johari and R. M. Shanker, J. Pharm. Sci., 2008, 97, 3233.

18 E. Tombari, C. Ferrari, G. P. Johari and R. M. Shanker, J. Phys. Chem. B, 2008, 112, 10806.

19 V. Caron, C. Bhugra and M. J. Pikal, J. Pharm. Sci., 2010, 99, 3887.

20 A. M. Triggle, E. Shefter and D. J. Triggle, J. Med. Chem., 1980, 23, 1442 .
21 K. L. A. Chan, O. S. Fleming, S. G. Kazarian, D. Vassou, G. D. Chryssikos and V. Gionis, J. Raman Spectrosc., 2004, 35, 353.

22 H. Wagner and R. Richert, J. Phys. Chem. B, 1999, 103, 4071.

23 M. R. Caira, Y. Robbertse, J. J. Bergh, M. N. Song and M. M. De Villiers, J. Pharm. Sci., 2003, 92, 2519.

24 M. Klimakow, J. Leiterer, J. Kneipp, E. Rossler, U. Panne, K. Rademann and F. Emmerling, Langmuir, 2010, 26, 11233.

25 E. Hempel, H. Huth and M. Beiner, Thermochim. Acta, 2003, 403, 105.

26 A. A. Decroix, L. Carpentier and M. Descamps, Philos. Mag., 2008, 88, 3925.

27 R. Kahlau, T. Gnutzmann, F. Emmerling, K. Rademann and E. A. Rossler, J. Chem. Phys., 2012, 137, 054505.

28 A. Kudlik, S. Benkhof, T. Blochowicz, C. Tschirwitz and E. Rossler, J. Mol. Struct., 1999, 479, 201.

29 C. Gainaru, R. Kahlau, E. A. Roessler and R. Boehmer, J. Chem. Phys., 2009, 131, 184510.

30 R. Kahlau, D. Kruk, T. Blochowicz, V. N. Novikov and E. A. Roessler, J. Phys.: Condens. Matter, 2010, 22, 365101. 